\title{
OUTER AUTOMORPHISMS OF SUPERSOLUBLE GROUPS
}

\author{
FEDERICO MENEGAZZO \\ Dipartimento di Matematica Pura e Applicata, Università di Padova, Via Belzoni 7, 35131 Padova, Italy \\ e-mail:menegazzo@math.unipd.it
}

ORAZIO PUGLISI

Dipartimento di Matematica, Università di Trento, via Sommarive, 38050 Povo, Italy

e-mail:puglisi@science.unitn.it

(Received 19 June, 1998)

to Derek Robinson on the occasion of his 60 th birthday

\begin{abstract}
In this paper we study the problem of the existence on non-inner automorphisms for the class of torsion-free supersolvable groups, answering a question raised by Robinson.
\end{abstract}

1991 Mathematics Subject Classification. 20F16, 20F28

Introduction. The famous, seminal papers by Gaschütz [2],[3] on the existence of outer automorphisms in finite $p$-groups have been both the conclusion of a long process and the starting point of a number of extensions and generalisations in different areas. The existence of non-inner automorphisms for infinite nilpotent $p$ groups was proved by Zalesski1 [9]; moreover, apart from the obvious exceptions, infinite nilpotent $p$-groups admit outer automorphisms of $p$-power order [6]. For general nilpotent groups the situation is quite different. In fact Zalesskil gives an example of a torsion-free nilpotent group, all of whose automorphisms are inner (see [10]) while, on the other hand, the existence of non-inner automorphisms had been proved by R. Ree [7] (but see also [1] and [4]) in the case of finitely generated, torsion free nilpotent groups. It is worth mentioning that it is still unknown whether an infinite finitely generated nilpotent group has an outer automorphism.

The problem of the existence of outer automorphisms has been addressed for other classes of groups and in this short note we will consider this question for torsion-free supersoluble groups. Our interest is motivated by a result proved by D.J.S. Robinson in [8]. In this paper the author shows that a torsion-free supersoluble group with trivial center always admits a non-inner automorphism. On this basis the following question was also asked by Robinson:

does every torsion-free supersoluble group have non-inner automorphisms?

In this note we show that the answer to the above question is negative, by constructing a class of torsion-free supersoluble groups, possessing only inner automorphisms.

An invariant which seems to play a decisive role in this context is the torsion-free rank of the abelian factor group $G / G^{\prime}$; we denote it by $\rho_{0}(G)$. We prove the following results.

(1) If $G$ is a torsion-free supersoluble group and $\rho_{0}(G) \geq 2$, then $G$ has non-inner automorphisms. 
(2) There are nontrivial torsion-free supersoluble groups $G$ with $\rho_{0}(G)=0$ such that $\operatorname{Aut}(G)=\operatorname{Inn}(G)$.

It is however not clear what happens when $\rho_{0}(G)=1$. Namely we have not been able to show that, if this is the case, the group $G$ admits non-inner automorphisms, nor could we provide examples of such groups all of whose automorphisms are inner.

In Section 1 we obtain, by elementary means, some sufficient conditions in order that a torsion-free supersoluble group admit non-inner automorphisms; these will imply our first result. Section 2 is devoted to the construction of a class of torsionfree supersoluble groups, without non-inner automorphisms, thus proving the second part of our statement.

1. General facts. We begin with an easy lemma.

Proposition 1. Let $G$ be a finitely generated torsion-free group. If $\operatorname{Aut}(G)=$ $\operatorname{Inn}(G)$, then

(1) $Z_{2}(G)=Z(G)$;

(2) $Z(G) \cap N=1$, for every $N \unlhd G$ with $G / N$ infinite cyclic.

Proof. (1) Suppose there is $u \in Z_{2}(G), u \notin Z(G)$. The subgroup $U=\langle u, Z(G)\rangle$ is abelian and normal in $G$, and the factor group $G / C_{G}(U)$ is a non-trivial, finitely generated, torsion-free abelian group. There is $K \unlhd G$ such that $C_{G}(U) \leq K$ and $G / K$ is infinite cyclic; moreover, $C_{G}(K) \leq K$. Choose $g \in G$ such that $G=\langle g, K\rangle$, and set $A=Z(K)$; as $A \geq Z(G) \neq 1$, we have $A>[g, A]$. For every $a \in A \backslash[g, A]$ the assignment

$$
\left\{\begin{array}{ll}
g & \mapsto g a \\
x & \mapsto x,
\end{array} \text { for every } x \in K\right.
$$

gives an automorphism of $G$ which is not inner: but this is a contradiction.

(2) Assume, by contradiction, that $1 \neq z \in N \cap Z(G)$. If $G=\langle g, N\rangle$, then the assignment

$$
\left\{\begin{array}{ll}
g & \mapsto g z \\
x & \mapsto x,
\end{array} \text { for every } x \in N,\right.
$$

defines a non-trivial central automorphism of $G$. By part (1), all such automorphisms are non-inner.

THEOREM 2. Let $G$ be a supersoluble torsion-free group such that $\operatorname{Aut}(G)=\operatorname{Inn}(G)$. If $G / G^{\prime}$ is infinite, then

(1) $Z(G)$ is infinite cyclic,

(2) $Z(G) \cap G^{\prime}=1$,

(3) $\rho_{0}(G)=1$.

Proof. In our case $G$ has a normal subgroup $N$ with infinite cyclic factor group $G / N$; of course, $G^{\prime} \leq N$. Moreover, $Z(G) \neq 1[\mathbf{8}]$. By Proposition $1,(2) Z(G) \cap N=1$, which implies (1) and (2). 
Suppose now that $\rho_{0}(G) \geq 2$. Then $G / Z(G) G^{\prime}$ is infinite, and there is a subgroup $N \geq Z(G) G^{\prime}$ such that $G / N$ is infinite cyclic. But this again contradicts Proposition $1,(2)$.

2. Examples. The following construction is in the spirit of some work of $\mathrm{H}$. Heineken [5].

THEOREM 3. For every integer $n \geq 5$ there is a supersoluble torsion-free group $G$ of Hirsch length $2 n$ and derived length 3 with $\operatorname{Aut}(G)=\operatorname{Inn}(G)$.

Proof. Let $A$ be the group given by the following generators and relations:

$$
\begin{aligned}
& A=\left\langle a_{1}, \ldots, a_{n}\right|\left[a_{i}, a_{j}, a_{k}\right]=1 \text { for all } i, j, k ; \\
& \left.\left[a_{i}, a_{j}\right]=1 \text { for all } i, j \text { such that } j \neq i+1, j \neq i-1\right\rangle
\end{aligned}
$$

$(n \geq 5$; here and later on, all indices are taken $\bmod n$, with representatives $1,2, \ldots, n)$.

$A$ is nilpotent of class 2 , torsion-free, and $A^{\prime}=Z(A)=\left\langle\left[a_{i}, a_{i+1}\right] \mid i=1, \ldots, n\right\rangle$ is free abelian.

Let $q_{1}, q_{2}, \ldots, q_{n}$ be distinct odd primes, and put $q=q_{1} \cdots q_{n}$. For $i=1, \ldots, n$ we define the following elements

$$
\begin{aligned}
y_{i} & =a_{i}^{q_{i-1} q_{i}}, \\
u_{i} & =\left[a_{i}, a_{i+1}\right]^{q_{i}}, \\
v_{i} & =a_{i}^{q_{i-1}} a_{i+1}^{2 q_{i+1}}\left[a_{i}, a_{i+1}\right]^{q_{i-1} q_{i+1}\left(q_{i}-1\right)} .
\end{aligned}
$$

It is easy to see that

$$
v_{i}^{q_{i}}=y_{i} y_{i+1}^{2}
$$

and to compute the relevant commutators:

$$
\begin{aligned}
& {\left[y_{i}, y_{i+1}\right]=u_{i}^{q_{i-1} q_{i} q_{i+1}},} \\
& {\left[v_{i}, y_{i-1}\right]=u_{i-1}^{-q_{i-2} q_{i-1}}, \quad\left[v_{i}, y_{i}\right]=u_{i}^{-2 q_{i-1} q_{i+1}}, \quad\left[v_{i}, y_{i+1}\right]=u_{i}^{q_{i-1} q_{i+1}},} \\
& {\left[v_{i}, y_{i+2}\right]=u_{i+1}^{2 q_{i+1} q_{i+2}}, \quad\left[v_{i}, v_{i+1}\right]=u_{i}^{q_{i-1}} u_{i+1}^{4 q_{i+2}}, \quad\left[v_{i}, v_{i+2}\right]=u_{i+1}^{2 q_{i+1}} .}
\end{aligned}
$$

The remaining commutators $\left[y_{i}, y_{j}\right],\left[v_{i}, y_{j}\right],\left[v_{i}, v_{j}\right]$ all vanish.

As a first step in the construction of the example we need a particular subgroup of $A$. Namely we set $F=\left\langle y_{1}, \ldots, y_{n}, u_{1}, \ldots, u_{n}, v_{1}, \ldots, v_{n}\right\rangle$. There are two subgroups of $F$ which will be of some importance for our aim. They are $U=\left\langle u_{1}, \ldots, u_{n}\right\rangle$, and $H=\left\langle y_{1}, \ldots, y_{n}, u_{1}, \ldots, u_{n}\right\rangle$. Notice that $U$ is central in $A$ (hence in $F$ ), $U$ is free abelian with basis $u_{1}, \ldots, u_{n}, F^{\prime} \leq U$ and $H / U$ is free abelian with basis $y_{1} U, \ldots, y_{n} U$.

We now derive some properties of $F$.

(1) $Z(F)=U$. 
Suppose first that $x \in H \cap Z(F), x=\left(\prod_{i} y_{i}^{t_{i}}\right) u$, for some $u \in U$. If some $t_{k} \neq 0$ then $\left[x, y_{k+1}\right]=\left[y_{k}^{t_{k}}, y_{k+1}\right]\left[y_{k+2}^{t_{k+2}}, y_{k+1}\right] \neq 1$. This shows that $H \cap Z(F) \leq U$. If now $x \in F \backslash H$, say $x \equiv \prod_{i} y_{i}^{r_{i}} \prod_{j} v_{j}^{s_{j}} \bmod U$ with $s_{k} \not \equiv 0 \bmod q_{k}$, we get

$$
x^{q} \equiv \prod_{l} y_{l}^{t_{l}} \bmod U, \text { where } t_{l}=\left(r_{l} q_{l} q_{l-1}+s_{l} q_{l-1}+2 s_{l-1} q_{l}\right)\left(q / q_{l} q_{l-1}\right)
$$

and so in particular $t_{k} \not \equiv 0 \bmod q_{k}$; hence $x^{q} \notin Z(F)$, and $x \notin Z(F)$, proving our claim.

(2) If $x \in F$ and $[x, F] \subseteq U^{2}$, then $x U$ is a square in $F / U$.

Again, suppose first that $x \in H, x \equiv \prod_{i} y_{i}^{t_{i}} \bmod U$. If $t_{k}$ is odd for some $k$, then

$$
\left[x, y_{k+1}\right]=\left[y_{k}, y_{k+1}\right]^{t_{k}}\left[y_{k+2}, y_{k+1}\right]^{t_{k+2}}=u_{k}^{t_{k} q_{k-1} q_{k} q_{k+1}} u_{k+1}^{-t_{k+2} q_{k} q_{k+1} q_{k+2}}
$$

is not a square in $U$ : hence (2) holds for elements of $H$. If now $x \in F$ and $[x, F] \subseteq U^{2}$, then $x^{q} \in H$ and $\left[x^{q}, F\right] \subseteq U^{2}$, so that $x^{q} U$ is a square in $F / U$; since $q$ is odd, $x U$ is a square too.

(3) $\left\langle y_{k} U\right\rangle$ is a pure subgroup of $F / U$, for $k=1, \ldots, n$.

Suppose that $x U=\prod_{i} y_{i}^{r_{i}} \prod_{j} v_{j}^{s_{j}} U$ and $(x U)^{m} \in\left\langle y_{k} U\right\rangle$, for some $m \neq 0$. Then $(x U)^{m q}=\prod_{l}\left(y_{l} U\right)^{t_{l} m} \in\left\langle y_{k} U\right\rangle$ with $t_{l}=\left(r_{l} q_{l} q_{l-1}+s_{l} q_{l-1}+2 s_{l-1} q_{l}\right)\left(q / q_{l} q_{l-1}\right)$ as above; so, for $l \neq k, r_{l} q_{l} q_{l-1}+s_{l} q_{l-1}+2 s_{l-1} q_{l}=0$. This implies $q_{l-1} \mid s_{l-1}$ and $q_{l} \mid s_{l}$, for every $l \neq k$; i.e. $x U \in H / U$. As we remarked above, $H / U=\left\langle y_{1} U\right\rangle \times \cdots \times\left\langle y_{n} U\right\rangle$; hence finally $x U \in\left\langle y_{k} U\right\rangle$.

(4) $[x, F]$ has rank 2 if and only if $U \neq\langle x U\rangle \leq\left\langle y_{i} U\right\rangle$, for some $i=1, \ldots, n$.

It is clear that, if $r \neq 0,\left[y_{i}^{r}, F\right]$ has rank 2. On the other hand, suppose $x U \notin\left\langle y_{i} U\right\rangle$, for $i=1, \ldots, n$; by (3) the same is true for $x^{q} U$, so that $x^{q} \equiv \prod_{i} y_{i}^{r_{i}} \bmod U$ with at least two non-zero exponents, say $r_{j}$ and $r_{k}(j \neq k)$. It is easy to check that, since $n \geq 5$, at least three of

$$
\left[x^{q}, y_{j-1}\right], \quad\left[x^{q}, y_{j+1}\right], \quad\left[x^{q}, y_{k-1}\right], \quad\left[x^{q}, y_{k+1}\right]
$$

are independent. Hence $\left[x^{q}, F\right]$ and $[x, F]$ have rank at least 3.

(5) $U,\left\langle y_{i}, U\right\rangle(i=1, \ldots, n)$ and $H$ are characteristic subgroups of $F$.

$U=Z(F)$ by (1); the set $\left\{\left\langle y_{1} U\right\rangle, \ldots,\left\langle y_{n} U\right\rangle\right\}$ is $\operatorname{Aut}(F)$-invariant by (4), which implies that also $H$ is characteristic. Now $\left[y_{i}, H\right]=\left\langle u_{i-1}^{q_{i-2} q_{i-1} q_{i}}, u_{i}^{q_{i-1} q_{i} q_{i+1}}\right\rangle$ has index $q_{i-2} q_{i-1}^{2} q_{i}^{2} q_{i+1}$ in its pure closure $\left\langle u_{i-1}, u_{i}\right\rangle$ in $U$. If $i \neq j$ these indices are different, which proves our claim. 
At this point, we notice that $A$ admits an automorphism $\sigma$ such that

$$
a_{i}^{\sigma}=a_{i}^{-1} \quad(i=1, \ldots, n) .
$$

It is obvious that $\sigma$ induces the identity on $A^{\prime}=Z(A)$ and that $\sigma^{2}=1$. Since $y_{i}^{\sigma}=y_{i}^{-1}$ and $u_{i}^{\sigma}=u_{i}$, we have $H^{\sigma}=H$. Moreover, the equality

$$
\begin{aligned}
v_{i} v_{i}^{\sigma} & =a_{i}^{q_{i-1}} a_{i+1}^{2 q_{i+1}}\left[a_{i}, a_{i+1}\right]^{q_{i-1} q_{i+1}\left(q_{i}-1\right)} a_{i}^{-q_{i-1}} a_{i+1}^{-2 q_{i+1}}\left[a_{i}, a_{i+1}\right]^{q_{i-1} q_{i+1}\left(q_{i}-1\right)}= \\
& =\left[a_{i}, a_{i+1}\right]^{2 q_{i-1} q_{i} q_{i+1}}=u_{i}^{2 q_{i-1} q_{i+1}}
\end{aligned}
$$

shows that $v_{i}^{\sigma}=v_{i}^{-1} u_{i}^{2 q_{i-1} q_{i+1}}$, so that $F^{\sigma}=F$.

(6) Put $b=\prod_{i=1}^{n-1}\left[y_{i}, y_{i+1}\right]$ and $L=\left\{x^{\sigma} x \mid x \in F\right\}$. Then $b \notin L$.

Since $x^{\sigma} \equiv x^{-1} \bmod U$ and for $u \in U(x u)^{\sigma} x u=x^{\sigma} x u^{2}$, the set $L$ is the union of some cosets of $U$ modulo $U^{2}$. Moreover, $q$ odd and $\left[x^{\sigma}, x\right]=1$ give $\left(x^{q}\right)^{\sigma} x^{q}=\left(x^{\sigma} x\right)^{q} \equiv x^{\sigma} x \bmod U^{2}$. It follows that if a coset $c U^{2} \subseteq L$ for any $c \in U$, then $c \equiv x^{\sigma} x \bmod U^{2}$, for some $x \in H$. Now let $x=\prod_{i} y_{i}^{t_{i}} u \in H$; we have

$$
\begin{aligned}
x^{\sigma} x & =y_{1}^{-t_{1}} \cdots y_{n}^{-t_{n}} y_{1}^{t_{1}} \cdots y_{n}^{t_{n}} u^{2}=\left(\prod_{1 \leq i<j \leq n}\left[y_{i}, y_{j}\right]^{t_{i} t_{j}}\right) u^{2}= \\
& =\prod_{i=1}^{n-1}\left[y_{i}, y_{i+1}\right]^{t_{i} t_{i+1}}\left[y_{n}, y_{1}\right]^{-t_{n} t_{1}} u^{2} .
\end{aligned}
$$

Suppose $b \equiv x^{\sigma} x \bmod U^{2}$ : since the set $\left\{\left[y_{i}, y_{i+1}\right] U^{2} \mid i=1, \ldots, n\right\}$ is a basis of $U / U^{2}$, this forces $t_{i} t_{n} \equiv 0 \bmod 2$; but then either $\left[y_{1}, y_{2}\right]$ or $\left[y_{n-1}, y_{n}\right]$ would be missing from $b$, a contradiction.

We can now complete our construction. Define $G=\langle F, s\rangle$, where $f^{s}=f^{\sigma}$, for all $f \in F$, and $s^{2}=b^{-1}$. It is clear that $Z(G)=Z(F)=U, G$ is supersoluble of Hirsch length $2 n, G$ has derived length 3 and $\rho_{0}(G)=0$. Moreover, $G$ is torsion-free: $F$ is torsion-free and, if some $s f(f \in F)$ is periodic, then $(s f)^{2}=1$; but this is impossible, since $(s f)^{2}=s^{2} f^{s} f=b^{-1} f^{\sigma} f$ and $b \notin L$.

Choose now $\alpha \in \operatorname{Aut}(G)$. The subgroup $F$ is the Fitting subgroup of $G$; hence $F$, $H, U,\left\langle y_{i}, U\right\rangle(i=1, \ldots, n)$ are characteristic in $G$. This implies that $y_{i}^{\alpha} U=y_{i}^{ \pm 1} U$, $(i=1, \ldots, n)$. Suppose that there is $i$ such that $y_{i}^{\alpha} U=y_{i} U$ and $y_{i+1}^{\alpha} U=y_{i+1}^{-1} U$. It is a consequence of the choice of $v_{i}$ that $y_{i} y_{i+1}^{2} U \in(F / U)^{q_{i}}$. Also $y_{i} y_{i+1}^{-2} U=$ $\left(y_{i} y_{i+1}^{2}\right)^{\alpha} U \in(F / U)^{q_{i}}$; hence $y_{i}^{2} U \in(F / U)^{q_{i}}$. This in turn, $q_{i}$ being odd, implies $y_{i} U \in(F / U)^{q_{i}}$, a contradiction to (3).

We have thus proved that $\alpha$ induces \pm 1 on $H / U$, hence also on $F / U$. After multiplication, if necessary, with the inner automorphism of $F$ induced by $s$, we may assume that $\alpha$ induces the identity on $F / U$; it then follows that $\alpha$ induces the identity also on $F^{\prime}$ and on $U$.

We now set $s^{\alpha}=s y$, where $y \in F$, and compute $[x, y]$ for an arbitrary $x \in F$. Notice that $s^{2}, x^{s} x,[x, \alpha],[x, y]$ are in $U$, so fixed by $\alpha$ and central in $G$. 


$$
\begin{aligned}
& \left(x^{s}\right)^{\alpha}=\left(s^{-1} x s\right)^{\alpha}=(s y)^{-1} x[x, \alpha] s y=x^{s y}[x, \alpha] ; \\
& x^{s} x=\left(x^{s} x\right)^{\alpha}=\left(x^{s}\right)^{\alpha} x[x, \alpha]=x^{s y}[x, \alpha]^{2} x ; \\
& {\left[y, x^{s}\right]=\left(x^{s y}\right)^{-1} x^{s}=[x, \alpha]^{2} ;} \\
& {[x, y]=[x, y]^{s}=\left[x^{s}, y^{-1}\right]=\left[y, x^{s}\right]=[x, \alpha]^{2}}
\end{aligned}
$$

since $s^{2}=\left(s^{2}\right)^{\alpha}=(s y)^{2}=s^{2} y^{s} y$ gives $y^{s}=y^{-1}$.

It follows from (2) that $y U=(w U)^{2}$, for some $w \in F$; hence $[x, \alpha]=[x, w]$, for all $x \in F$. If we multiply $\alpha$ by the inner automorphism induced by $w^{-1}$, we may assume that $\alpha$ is the identity on $F$ (and of course on $G / F$ which has order 2). Hence $\alpha$ comes from a derivation $G / F \rightarrow Z(F)$. Such derivations are in fact homomorphisms because $Z(F)=U=Z(G)$. However, $|G / F|=2$ and $U$ torsion-free imply that the only homomorphism $G / F \rightarrow U$ is the trivial one, which shows that $\alpha$ is the identity.

Acknowledgements. The authors wish to acknowledge partial support from Consiglio Nazionale delle Ricerche - G.N.S.A.G.A.

\section{REFERENCES}

1. G. Baumslag, Lecture notes on nilpotent groups, AMS Regional Conference series, Vol. 2 (1971).

2. W. Gaschütz, Kohomologische Trivialitäten und äussere Automorphismen von $p$ Gruppen, Math. Z. 88 (1965), 432-433.

3. W. Gaschütz, Nichtabelsche $p$-Gruppen besitzen äussere $p$-Automorphismen, $J$. Algebra 4 (1966), 1-2.

4. N. D. Gupta and S. E. Stonehewer, Outer automorphisms of finitely generated nilpotent groups, Arch. Math. (Basel) 31 (1978-79), 1-10.

5. H. Heineken, Automorphism groups of torsion-free nilpotent groups of class two in Symposia Mathematica, Vol. XVII (Convegno sui Gruppi Infiniti, INDAM, Roma, 1973), 235250.

6. F. Menegazzo and S. E. Stonehewer, On the automorphism group of a nilpotent $p$ group, J. London Math. Soc (2) 31 (1985), 272-276.

7. R. Ree, The existence of outer automorphisms of some groups, Proc. Amer. Math. Soc. 7 (1956), 962-964.

8. D. J. S. Robinson, Infinite soluble groups with no outer automorphisms, Rend. Sem. Mat. Univ. Padova 62 (1980), 281-294.

9. A. E. Zalesskiŭ, A nilpotent p-group has an outer automorphism, Dokl. Akad. Nauk SSSR 196 (1971); Soviet Math Dokl. 12 (1971), 227-230.

10. A. E. Zalesskiŭ, An example of a torsion-free nilpotent group having no outer automorphisms, Mat. Zametki 11 (1972), 21-26; Math. Notes 11 (1972), 16-19. 